\title{
BMJ Open Protocol for a scoping review of digital health for older adults with cancer and their families
}

\author{
Karis Kin-Fong Cheng (D) , ${ }^{1}$ Rosalind Chiew-Jiat Siah, ${ }^{1}$ Emma Ream, ${ }^{2}$ \\ Ravindran Kanesvaran, ${ }^{3}$ Jo Armes $^{2}$
}

To cite: Cheng KK-F, Siah RC-J, Ream E, et al. Protocol for a scoping review of digital health for older adults with cancer and their families. BMJ Open 2020;10:e038876. doi:10.1136/ bmjopen-2020-038876

- Prepublication history and additional material for this paper are available online. To view these files, please visit the journal online (http://dx.doi org/10.1136/bmjopen-2020038876).

Received 28 March 2020 Revised 23 June 2020 Accepted 07 July 2020

\section{Check for updates}

C Author(s) (or their employer(s)) 2020. Re-use permitted under CC BY-NC. No commercial re-use. See rights and permissions. Published by BMJ.

${ }^{1}$ Alice Lee Centre for Nursing Studies, Yong Loo Lin School of Medicine, National University of Singapore, Singapore

${ }^{2}$ School of Health Sciences, Faculty of Health and Medical Sciences, University of Surrey, Guildford, UK

${ }^{3}$ Division of Medical Oncology, National Cancer Centre

Singapore, Singapore

Correspondence to

Professor Karis Kin-Fong Cheng; nurckfk@nus.edu.sg

\section{ABSTRACT}

Introduction The potential for digital medicine and healthcare in geriatric oncology settings has received much attention. This scoping review will summarise the nature and extent of the existing literature that describes and examines digital health development, implementation, evaluation, outcome and experience for older adults with cancer, their families and their healthcare providers.

Methods and analysis Arksey and 0'Malley's six stages of scoping review methodology framework will be used. Searches will be conducted in Cochrane Central Register of Controlled Trials (CENTRAL), PubMed, Embase via OvidSP, Cumulative Index to Nursing and Allied Health Literature (CINAHL) Plus via EBSCO, Scopus and PsycINFO via OvidSP for published articles in peer-reviewed scientific journals from year 2000 onwards. In addition, we will screen databases for all prospectively registered trials. Research articles using quantitative or qualitative study design or reviews will be included if they describe or report the design, development or usability of digital health interventions in the treatment and care of patients 65 years of age or older with cancer and their families before, during and after cancer treatment. Grey literature will not be searched and included. Two investigators will independently perform the literature search, eligibility assessments and study selection. A Preferred Reporting Items for Systematic Reviews and Meta-Analyses flow diagram for the scoping reviews (PRISMA-ScR) will be used to delineate the search decision process. For included articles, the extracted results will be synthesised both quantitatively and qualitatively and reported under key conceptual categories of this scoping review. Research gaps and opportunities will be identified and summarised. Ethics and dissemination Since this review will only include published data, ethics approval will not be sought. The results of the review will be published in peerreviewed scientific journals. We will also engage with relevant stakeholders within research team's networks to determine suitable approaches for dissemination.

\section{INTRODUCTION}

More than $60 \%$ of all cancers arise in the older adult population. ${ }^{1}$ It is estimated by 2030 , people 65 years of age or older will account for $70 \%$ of cancer diagnoses. ${ }^{2}$ Coupled with an increased incidence of cancer among older people is the unprecedented demographic

\section{Strengths and limitations of this study}

- This review will use an established, rigorous and systematic framework for conducting scoping reviews to explore in detail the current state of knowledge and evidence about digital health for older adults with cancer and their families.

- Gaining insights into the characteristics and contexts leading to positive outcomes, patient/family and healthcare provider experience and integration challenges and barriers will provide a big picture on digital health progress in geriatric oncology settings and would be helpful in identifying what recommendations can be made to improve gaps in the research and knowledge base.

- This scoping review will not examine health economic and clinical use evidence for digital health.

- We will not assess the risk of bias and the quality of evidence of included studies as the goals are to identify and summarise research gaps and opportunities.

- Given the anticipated volume of peer-reviewed scientific literature and databases for prospectively registered trials, grey literature will not be searched and included.

growth of the elderly population. The United Nations highlighted that populations are becoming older in all regions of the world, with an estimate that one in six people globally will be 65 years or older by $2050 .{ }^{3}$ Such epidemiological evidence highlights the importance of advancing the knowledge base in geriatric-oncology settings and improving clinical management. Older people make up a unique group of patients in a clinical cancer setting and are at increased risk for toxic side effects during cancer treatment. ${ }^{45}$ Treatment of older patients is complicated by the fact that many are entering the cancer trajectory with pre-existing comorbidities and susceptibility to the progressive accumulation of multiple chronic diseases and a decline in functional capabilities. $^{6-9}$ The assessment, treatment and supportive care needs of older patients with cancer are substantial and complex. 
Geriatric oncology has progressed immensely in terms of understanding the predicative ability of baseline comprehensive geriatric assessment for chemotherapy toxicity and treatment completion. ${ }^{810}$ However, concerns remain about how to improve the experience of older cancer patients ${ }^{11}$ and their tolerance of and adherence to cancer treatment regimens to optimise the full benefits of chemotherapy. ${ }^{12}$ Chouliara et al indicated that symptom management, chemotherapy, choices of medical provider, complementary treatments and family involvement in the patient's care are important decisionmaking topics for older people with cancer and their relatives. ${ }^{13}$ There is a pressing need to open new avenues for effective treatment, care and support of older patients with cancer to optimise clinical and patient outcomes.

The growth and advancement of digital technology through the integration of sensor arrays, interactive multitouch screens, voice and video media, interactive longitudinal data, the advanced functions of apps, high computing power and fast network speed have revolutionised the medical and healthcare sciences. Shen and Naeim highlighted that digital technology has transformed the ways that healthcare can be delivered all across the world. ${ }^{14}$ Literature resources are rapidly expanding to include digital health applications and evaluations, technology integration into healthcare systems, technology literacy and exposure and technology acceptance, barriers and engagement across a wide spectrum of healthcare domains. ${ }^{15-17}$ These insights are helpful for the geriatric oncology field.

Technology use by older adults is increasing, and the potential for digital medicine and healthcare in oncology settings has received much attention. In 2016, the adoption rates of smartphones and tablets by older adults 60 to 69 years of age were $46 \%$ and $41 \%$, respectively. ${ }^{18}$ Anderson and Perrin indicated that $67 \%$ of individuals older than 65 years of age have Internet access and go online. ${ }^{19}$ Recent reviews identified the potential of, and need for, both remote digital self-reporting solutions for cancer and treatment-related symptoms using web-based or smartphone-based portals, and Internet of Thingsbased solutions using various ambient-sensing technologies to enable objective and real-time monitoring of treatment toxicity, symptoms and functional status in geriatric oncology settings. ${ }^{14} 20$ Denis et al demonstrated that remote symptom monitoring using a web-based selfreport of symptoms improved overall survival rates among lung cancer patients 35.7 to 88.1 years of age (median age, 64.5 years)..$^{21}$ The functional performance status of older cancer patients has also been monitored with wearable electronic activity monitoring technologies. ${ }^{22}$ Villani et al evaluated eHealth stress inoculation training intervention on emotional regulation and cancer-related well-being in 29 women over 55 years of age with breast cancer (mean age, 62.76 years), and revealed a good level of acceptance of the eHealth intervention, an increase in relaxation and a reduction in anxiety among women in the intervention group. ${ }^{23}$ Hoogland et al surveyed eHealth literacy in older adults with cancer and found that older adults had significantly lower eHealth literacy than younger patients, which suggests older cancer patients' needs and abilities should be considered when designing and implementing health information technology. ${ }^{17}$

There is a need for comprehensive evidence to inform the research and development of digital health solutions, and to understand the imperatives of designing, implementing and evaluating digital healthcare initiatives in the context of geriatric oncology populations. Peters et al noted that a scoping review is particularly useful when a body of literature has not been comprehensively reviewed. ${ }^{24}$ Scoping reviews are one of the most common forms of review in healthcare sciences, and are characterised as a broader approach with the aim of mapping literature and addressing a broader research question. ${ }^{24}$ An appealing feature of scoping review methodology, contrary to other review approaches, is that it does not limit the parameters of the review to randomised controlled trials or require methodological homogeneity of studies included in a review. ${ }^{25}$ Such an approach is consistent with literature that the hierarchies of evidence used in existing health research should be replaced by embracing diverse research methods approaches. ${ }^{26}$ Indeed, the inclusion of published literature in a particular field as a whole regardless of its methodological approaches, settings and contexts is necessary to provide a big picture of existing knowledge, thereby improving research planning, strategic research prioritisation and evidenceinformed policies. ${ }^{27}$ Literature also indicates that scoping reviews are rigorous in their approach to providing rich and contextual details evidence of research activity in a particular area. ${ }^{28}$ Despite the strengths, the lack of methodological quality assessment and quality of evidence grading even for empirical evidence minimises the ability of results from a scoping review to inform clinical decision-making. A recent critical review of the scoping review methods indicated that results of scoping reviews are not used to create recommendations for policy or practice. ${ }^{28}$ Other important challenges in scoping reviews are the level of complexity across multiple stages ${ }^{28}$ and practical issues related to the large corpus and the wide range of research literature. ${ }^{25}$ Therefore, it is crucial to follow a sound methodological guidance or framework, ${ }^{28}$ and to have a balance between breadth, comprehensiveness and feasibility. ${ }^{29}$ Consistent with the rationale for conducting scoping reviews, the scoping review methodology of knowledge synthesis is well suited to the present review study purpose. Here we describe a scoping review protocol to systematically review published literature on digital health for older adults with cancer, their families and their healthcare providers.

The purpose of this scoping review protocol is to systematically map and explore the literature and evidence describing and examining digital health development, implementation, evaluation, outcome and experience for older adults with cancer, their families and their healthcare providers. We focus primarily on 
evaluation of baseline performance status and/or geriatric assessment, clinical assessment, treatment outcomes and patient management including self-management and self-monitoring. The following points in the cancer pathway are considered: before, during and after cancer treatment, which includes surgery, adjuvant chemotherapy and/or radiotherapy, targeted therapy, follow-up care during remission and survivorship care. Our goals are to direct future research efforts by identifying gaps and limitations in the literature and to highlight relevant determinants of positive outcomes in the emerging field of geriatric oncology.

\section{METHODS}

Our protocol was developed using the scoping review methodological framework proposed by Arksey and O'Malley $^{30}$ and further refined by the Joanna Briggs Institute. ${ }^{24}$ The approach describes six methodological stages: (1) identification of the research question, (2) identification of relevant studies, (3) selection of studies, (4) extracting and charting the results, (5) collating, summarising and reporting the results and (6) consultation with stakeholders (optional).

\section{Identifying the research questions (stage 1)}

This scoping review will answer the following questions:

- To what extent digital health has been established or used in the field of geriatric oncology?

- What scientific evidence exists for the effectiveness of various types of digital health interventions for older cancer patients and their families?

- Which participant and digital health intervention characteristics and context are associated with positive outcomes?

- What are the experiences of older cancer patients, their families and their healthcare providers of digital healthcare?

- What are challenges and barriers of integrating digital health into clinical practice?

\section{Identification of relevant studies (stage 2) \\ Search strategy}

The team collaborated to plan a search strategy in consultation with a medical librarian to identify a comprehensive list of relevant literature specific to digital health for older adults with cancer and their families. The electronic search for literature will focus on retrieving published articles in peer-reviewed scientific journals by a systematic search of the following databases: Cochrane Central Register of Controlled Trials (CENTRAL), PubMed, Embase via OvidSP, Cumulative Index to Nursing and Allied Health Literature (CINAHL) Plus via EBSCO, Scopus and PsycINFO via OvidSP.

We will retrieve all journal articles published in the English language from year 2000 onwards (1 January 2000 to 25 May 2020) to accommodate the emergence of the geriatric oncology as a field with the formation of
International Society of Geriatric Oncology in $2000,{ }^{31}$ hence research in this field only became mainstream and gained momentum after 2000. In addition, the publishing date range (within the last 20 years) will accommodate the wide adoption of digital healthcare following publication of WHO vision for digital health ${ }^{32}$ and after the introduction of smartphones. Two investigators will independently perform the literature search, eligibility assessments and study selection. We performed a pilot search on PubMed to identify relevant keywords contained in the title, abstract and subject descriptors. We will use the following search terms related to digital health and older adults with cancer, with various combinations in each electronic database while using controlled vocabulary with the Boolean operators AND and OR. We will use appropriate subject headings (eg, MedicalSubject Headings) whenever possible. The term 'healthcare providers' encompasses oncologists, nurses, nurse practitioners, clinicians, physicians, general practitioners and pharmacists in this scoping review. A copy of the search strategies and the preliminary searches results in each electronic database is included as an online supplementary file.

The basic search terms used will be:

1. (Older adult* OR Older person OR Elderly OR Geriatric) AND (Neoplasm* OR Cancer OR Carcinoma)

2. (Famil* OR Carer* OR Caregiver* OR Health care provider* OR Healthcare professional* OR Nurs* OR Oncolog* OR General practitioner OR Practitioner OR Nurse practitioner OR Pharmacy OR Clinician OR Physician)

3. (Internet OR Telemonitor* OR Teleconsultat* OR Telehealth OR Telecare OR Website* OR App OR Apps OR Application* OR Digital OR eHealth OR mHealth)

4. (1 AND 2) AND 3

We will also screen reference lists of all identified studies and reviews for any relevant publications. Finally, we will screen databases for ongoing clinical trials and all prospectively registered trials, including the WHO International Clinical Trials Registry Platform and ClinicalTrials.gov.

\section{Selection of studies (stage 3 )}

Studies will be selected following two stages of screening. The first stage will be an initial screening of titles and abstracts by two reviewers independently to assess relevance. The initial review will be done independently, and the reviewers will discuss the results once screening is complete. Resolving disagreements will be attempted first by the two reviewers, but if necessary, a third member of the research team will be consulted to reach consensus. Once the initial decision is reached on which articles to include, we will begin the second stage of conducting a full-text review. Two reviewers will independently assess the articles to determine whether they meet the inclusion criteria. Disagreements regarding inclusion will be discussed and resolved by consensus, with a third 
Table 1 Inclusion and exclusion criteria

\section{Inclusion criteria}

Population: families, oncology healthcare providers
Older adults with cancer, their

Older individuals 65 years of age or older (or where the average age of the study sample was 65 years of age or older) who had any cancer diagnosis.

Family members including carers of older adults with cancer, and/or oncology healthcare providers.

Concept or Focus:

Development, usability, outcomes, effectiveness, context and experiences of digital health interventions; and challenges and barriers of integrating digital health into clinical practice

\section{Studies describing or reporting the} design, development or usability of digital health interventions (teleconsultation, telemedicine, telehealth, telecare, telemonitoring, geriatric and oncology care programmes, patient platforms, self-care websites, informative websites and mobile applications) in the treatment and care of older patients with cancer and their families before, during and after cancer treatment (surgery, adjuvant chemotherapy and/or radiotherapy, targeted therapy) including follow-up care during remission and survivorship care .

Studies evaluating the effectiveness of various types of digital health interventions for older cancer patients and their families, and the characteristics and contexts contributing to positive clinical and patient outcomes or experiences.

Studies reporting the challenges and barriers of integrating digital health into clinical practice in a geriatric oncology setting.

\section{Context:}

Provision of geriatric oncology treatment including surgery, adjuvant chemotherapy and/or radiotherapy, targeted therapy, services and care in clinical and/or community settings Others
Studies conducted in clinical and/or community (including home) settings.

\section{Exclusion criteria}

Studies on digital health interventions of adults with cancer where 65 years of age or older were not reported separately.

Studies reporting costs of the digital health interventions only.

Studies on digital health interventions being used for diagnostics/imaging

\section{Studies conducted in screening and} diagnostic settings.

\section{English}

Articles published after 2000

Original or review research articles

Qualitative (qualitative descriptive, phenomenological, ethnographical, grounded theory, realistic evaluation, action research, and so on), quantitative (random controlled trials, cohort study, case-control and cross-sectional, and so on) or reviews (systematic review, meta-analysis, scoping review, narrative review, and so on) or descriptions of study protocols

\section{Editorials \\ Opinion/perspective papers \\ Conference abstracts \\ Case reports \\ Theses/dissertations.}

member of the research team adjudicating articles without a consensus agreement or with questions about their relevance or eligibility. The first stage of screening is underway.

Table 1 delineates the inclusion and exclusion criteria, following the Population, Concept and Context categories for scoping reviews. ${ }^{24}$ We will iteratively refine the inclusion and exclusion criteria to align potentially eligible studies to the purpose of this scoping review. ${ }^{24}$ Inclusion criteria are studies that focus on: (1) older individuals 65 years of age or older (or where the mean or median age of the study sample was 65 years of age or older) who had 
any cancer diagnosis, and/or family members including carers of older adults with cancer, and/or healthcare providers; (2a) describing or reporting the design, development or usability of digital health interventions in the treatment and care of older patients with cancer and their families before, during and after cancer treatment; (2b) evaluating the effectiveness of various types of digital health interventions for older cancer patients and their families, and the characteristics and contexts contributing to positive clinical and patient outcomes or experiences; (2c) reporting the challenges and barriers of integrating digital health into clinical practice in a geriatric oncology setting; and (3) how geriatric oncology treatment, services and care are provided in clinical and community settings. The inclusion of both clinical and community settings is meant to provide a comprehensive picture of the digital health landscape in geriatric oncology settings, particularly gaining insights into the interface between clinical treatment/care in hospital and remote monitoring of treatment toxicity, symptoms and functional status in community. Research articles using quantitative or qualitative study design or reviews will be included to support the greater breadth of this scoping review. ${ }^{24}$ Peer-reviewed scientific articles describing study protocols will also be included.

A Preferred Reporting Items for Systematic Reviews and Meta-Analyses flow diagram for the scoping reviews (PRISMA-ScR) will be used to delineate the search decision process for the scoping review. ${ }^{33}$ This will include the results from the search, removal of duplicate citations, study selection, full retrieval and additions from reference list searching and final selection for inclusion in the scoping review.

\section{Extracting and charting the results (stage 4)}

In this stage, articles that meet the criteria for inclusion will be summarised. Literature highlighted that the essence of data extraction is to record characteristics of the included studies and key information relevant to the review questions. ${ }^{24}$ Congruent with the purpose and questions of this scoping review and taking reference from the existing digital health interventions design and evaluation framework,${ }^{34}$ we identified a priori categories and related variables including 'general information categories', 'key conceptual categories' and 'additional categories' with related variables described below as the data extraction framework to guide the extraction and charting of data from the included studies. A data chart will be developed based on the data extraction framework by our research team. The general information and the key quantitative and/or qualitative findings of the included studies will be extracted into the data chart. The data chart will be piloted by two reviewers on two or three studies, and differences in charting will be resolved by a third member of the research team. After the pilot trial, the results will be discussed with the research team to determine whether the data chart satisfactorily captures the information to align and be consistent with the purpose and questions of this scoping review. ${ }^{35}$ Refinements will be made after the pilot trial if deemed necessary by the research team. In addition, the data extraction framework will be updated or refined according to categories and related variables emerging as the conduct of the review progresses. ${ }^{24}$ Any iterative changes or refinements needed during the actual conduct of the full review will be clearly detailed and explained in the future scoping review report paper(s).

\section{General information categories}

General information that will be included in this chart include: description of study characteristics (eg, year of publication, country of origin, the geographical location in which the research was conducted, aims/ purpose, methodology and sample size); descriptions of study populations (eg, age, gender, residence, ethnicity, cultural background, socioeconomic status, functionality, cognition, disability, comorbidity and family carers); type and stage of cancer, and years of survival after cancer diagnosis; milestones along the cancer continuum (eg, before, during and after cancer treatment including follow-up care during remission and survivorship care); cancer treatment (eg, surgery, chemotherapy, radiation and/or targeted therapy); context of cancer treatment and care (eg, clinical and community); and survival from diagnosis. For studies in which digital health interventions are developed, the theoretical basis underpinning the intervention, and the extent to which stakeholders including patients, family and healthcare providers were involved in the development of the intervention will be extracted when possible. For studies that evaluate digital health interventions, the digital health intervention type according to WHO classification ${ }^{36}$ and comparator, mode of delivery, study design, outcome measures and context and key findings that relate to the scoping review questions will be extracted.

\section{Key conceptual categories}

An initial set of data extraction variables that correspond with the key conceptual categories of this scoping review will be extracted, including the development (eg, conceptual and technological foundation; intervention component and content), usability (eg, usage rate), effectiveness (eg, process, impact and outcome), context (eg, personal and environment context) and experiences of digital health interventions (eg, positive, neutral and negative experience); and the challenges and barriers (eg, individual characteristics, social support, workforce, planning, funding for equipment, cost of technology, guidelines, methodology and ways and timing in which digital health could be integrated/adapted) of integrating digital health into clinical practice. Additionally, methodological and knowledge gaps in the research and future research directions offered by author(s) will be extracted from the included studies. This information will also be noted for studies whose study population was not older adults with cancer, but rather families or healthcare providers. 


\section{Additional categories}

We drew on digital health intervention design and evaluation framework $^{34}$ to identify the following sets of variables which will be extracted from the included studies to strengthen the technological foundation of evidence: eHealth literacy, ease of use, perceived benefit, content quality, personalisation, adherence, safety, privacy and security.

\section{Collating, summarising and reporting the results (stage 5) Describing and reporting the study characteristics}

The extracted data will be summarised to provide a description of the collected data. Descriptive statistics including frequencies and central measures of tendency will be used to report the number of studies under each general information, key conceptual and additional category, such as the type of study design, the type of digital health intervention, the outcomes or experiences of digital healthcare at each stage of the cancer continuum, the type of contributing characteristics and the type of challenges and barriers. Descriptive statistics and numerical summaries will be reported in tables and/or in narrative form.

\section{Synthesising and reporting qualitative and quantitative evidence}

The extracted results will be synthesised and reported under key conceptual categories aligning to the purpose and questions of this scoping review including: the development, usability, outcomes, effectiveness, context and experiences of digital health interventions; and the challenges and barriers of integrating digital health into clinical practice. In accordance with recommendations from the literature, ${ }^{37}$ a parallel-results convergent synthesis design will be used to synthesise quantitative and qualitative data, where both types of evidence will be analysed and presented separately, with integration occurring during the interpretation of results. ${ }^{37}$ A particular strength of the parallel-results convergent design is that it provides a synthesis strategy for addressing multiple complementary review questions pertaining to our broadly covered topic with regards to digital health for older adults with cancer and their families, thereby providing a big picture of existing knowledge, evidence and research gaps. For quantitative data, we will use frequency distribution analysis to describe the data and map out the evidence by providing a summary of the counts of the included studies. ${ }^{29}$ We will also use thematic synthesis to contextualise the findings of the quantitative studies where appropriate. For qualitative data, a narrative synthesis of the findings will be conducted for review questions pertaining to development, usability, outcomes and effectiveness. ${ }^{37}$ Major review findings will be summarised and explained. In addition, thematic synthesis will be used to synthesise data from qualitative studies that address the review questions related to context and people's views and experiences. Major themes will be identified and developed across included studies. ${ }^{37}$ Research gaps and opportunities will also be identified and summarised. The review results may be presented as a 'numerical summary', 'narrative summary', 'table', 'conceptual map' and/or 'schematic representation' of the data. We may decide on additional presentation formats after data extraction from the included studies, so to make sure results data are clear and visually compelling to the readers. ${ }^{28}$

\section{Consultation with stakeholders (stage 6)}

We will engage with relevant stakeholders within the research team's networks, including oncology/geriatric oncology healthcare providers, regional and international community and professional organisations and digital health developers, if it is feasible for them to contribute towards the interpretation as well as research and policy implications of the results, so as to improve the relevance and impact of this scoping review. In addition, we aim to engage with stakeholders to determine suitable approaches for dissemination and additional knowledge translation initiatives, so as to optimise knowledge translation.

\section{PATIENT AND PUBLIC INVOLVEMENT}

Patient and public involvement strategy or group was not used in the development of this scoping review protocol. Neither patients nor the public were involved in the design and the development of the protocol. Nevertheless, we might invite older adults with cancer and their families from patient support groups within research team's networks at the consultation phase to determine suitable approaches for dissemination of review findings.

\section{ETHICS AND DISSEMINATION}

This scoping review protocol outlines a method to rigorously and systematically search and map the literature on digital health for older adults with cancer and their families. Since this review will only include published data, ethics approval will not be sought. Results from this review will be disseminated through peer-reviewed scientific journals and major professional conferences. We will also engage with relevant stakeholders within research team's networks to determine suitable approaches for dissemination after the completion of stage five of the review methodology. We anticipate that our review results regarding the current state of knowledge and evidence about digital health in geriatric oncology could provide direction for future research efforts and inform clinical practice and policy. Congruent with the scoping review methodology guidance, we will consider the implications of review results within the broader context. ${ }^{25}$

Acknowledgements The authors would like to thank Ms Wong Suei Nee, Medical Librarian, for her fantastic assistance in search strategy planning.

Contributors KK-FC and JA contributed to the conception or design of the review. KK-FC drafted the review protocol with suggestions from RK, ER and JA. KK-FC and $\mathrm{RS}$ constructed the search, and RS performed a preliminary search. KK-FC, RS, ER, RK and JA provided final approval of the review protocol. 
Funding The authors have not declared a specific grant for this research from any funding agency in the public, commercial or not-for-profit sectors.

Competing interests None declared.

Patient consent for publication Not required.

Provenance and peer review Not commissioned; externally peer reviewed.

Open access This is an open access article distributed in accordance with the Creative Commons Attribution Non Commercial (CC BY-NC 4.0) license, which permits others to distribute, remix, adapt, build upon this work non-commercially, and license their derivative works on different terms, provided the original work is properly cited, appropriate credit is given, any changes made indicated, and the use is non-commercial. See: http://creativecommons.org/licenses/by-nc/4.0/.

ORCID iD

Karis Kin-Fong Cheng http://orcid.org/0000-0002-4760-4771

\section{REFERENCES}

1 Cinar D, Tas D. Cancer in the elderly. North Clin Istanb 2015;2:73-80.

2 Smith BD, Smith GL, Hurria A, et al. Future of cancer incidence in the United States: burdens upon an aging, changing nation. JCO 2009;27:2758-65.

3 Department of Economic, Social Affairs Population Division. United nations. 2019. world population aging,, 2019. Available: https://www. un.org/en/development/desa/population/publications/pdf/ageing/ WorldPopulationAgeing2019-Highlights.pdf [Accessed 10 Feb 2020].

4 Mohile S, Dale W, Hurria A. Geriatric oncology research to improve clinical care. Nat Rev Clin Oncol 2012;9:571-8

5 Mohile SG, Dale W, Somerfield MR, et al. Practical assessment and management of vulnerabilities in older patients receiving chemotherapy: ASCO guideline for geriatric oncology. $J$ Clin Oncol 2018;36:2326-47.

6 Cheng KKF, Lee DTF. Effects of pain, fatigue, insomnia, and mood disturbance on functional status and quality of life of elderly patients with cancer. Crit Rev Oncol Hematol 2011;78:127-37.

7 Koll T, Pergolotti M, Holmes HM, et al. Supportive care in older adults with cancer: across the continuum. Curr Oncol Rep 2016;18:51.

8 Hurria A, Mohile S, Gajra A, et al. Validation of a prediction tool for chemotherapy toxicity in older adults with cancer. J Clin Oncol 2016;34:2366-71.

9 Naeim A, Aapro M, Subbarao R, et al. Supportive care considerations for older adults with cancer. J Clin Oncol 2014;32:2627-34.

10 Johnson M. Chemotherapy treatment decision making by professionals and older patients with cancer: a narrative review of the literature. Eur J Cancer Care 2012;21:3-9.

11 Farrington N, Richardson A, Bridges J. Interventions for older people having cancer treatment: a scoping review. J Geriatr Oncol 2020;11:769-83.

12 Boulding W, Glickman SW, Manary MP, et al. Relationship between patient satisfaction with inpatient care and hospital readmission within 30 days. Am J Manag Care 2011;17:41-8.

13 Chouliara Z, Kearney N, Stott D, et al. Perceptions of older people with cancer of information, decision making and treatment: a systematic review of selected literature. Ann Oncol 2004;15:1596-602.

14 Shen J, Naeim A. Telehealth in older adults with cancer in the United States: the emerging use of wearable sensors. J Geriatr Oncol 2017;8:437-42.

15 Garg S, Williams NL, Ip A, et al. Clinical integration of digital solutions in health care: an overview of the current landscape of digital technologies in cancer care. JCO Clin Cancer Inform 2018;2:1-9.
16 Gulliford M, Alageel S. Digital health intervention at older ages. The Lancet 2019;1.

17 Hoogland Al, Mansfield J, Lafranchise EA, et al. eHealth literacy in older adults with cancer. J Geriatr Oncol 2020;11:1020-2.

18 Rainie L, Perrin A. Technology adoption by baby boomers (and everybody else. Washington, DC: Pew Research Center, 2016. https://www.pewresearch.org/internet/2016/03/22/technologyadoption-by-baby-boomers-and-everybody-else/

19 Anderson M, Perrin A. Tech adoption climbs among older adults. Pew research center, 2017. Available: www.pewresearch.org/ internet/2017/05/17/technology-use-among-seniors/ [Accessed 13 Aug 2020].

20 Fallahzadeh R, Rokni SA, Ghasemzadeh $\mathrm{H}$, et al. Digital health for geriatric oncology. JCO Clin Cancer Inform 2018;2:1-12.

21 Denis F, Lethrosne C, Pourel N, et al. Randomized trial comparing a Web-mediated follow-up with routine surveillance in lung cancer patients. J Nat/ Cancer Inst 2017;109:jnci/djx029.

22 Kelly CM, Shahrokni A. From shelf to bedside-wearable electronic activity monitoring technologies might assist oncologists in functional performance status assessment of older cancer patients. Clin Colorectal Cancer 2016;30:S1533-0028.

23 Villani D, Cognetta C, Repetto C, et al. Promoting emotional well-being in older breast cancer patients: results from an eHealth intervention. Front Psychol 2018;9:2279.

24 Peters MDJ, Godfrey CM, Khalil H, et al. Guidance for conducting systematic scoping reviews. Int J Evid Based Healthc 2015;13:141-6.

25 Levac D, Colquhoun H, O'Brien KK. Scoping studies: advancing the methodology. Implement Sci 2010;5:69.

26 Rawlins M. De Testimonio: on the evidence for decisions about the use of therapeutic interventions. Clin Med 2008;8:579-88.

27 Nyanchoka L, Tudur-Smith C, Thu VN, et al. A scoping review describes methods used to identify, prioritize and display gaps in health research. J Clin Epidemiol 2019;109:99-110.

28 Lockwood C, Dos Santos KB, Pap R. Practical guidance for knowledge synthesis: Scoping review methods. Asian Nurs Res 2019;13:287-94.

29 Ghalibaf AK, Nazari E, Gholian-Aval M, et al. Comprehensive overview of computer-based health information tailoring: a scoping review protocol. BMJ Open 2017;7:e019215.

30 Arksey H, O'Malley L. Scoping studies: towards a methodological framework. Int J Soc Res Methodol 2005;8:19-32.

31 SIOG. International Society of geriatric oncology. Available: https:// www.siog.org/

32 World Health Organization. WHA58.28 eHealth. Available: https:// www.who.int/healthacademy/media/WHA58-28-en.pdf?ua $=1$ [Accessed 11 Jun 2020].

33 Tricco AC, Lillie E, Zarin W, et al. PRISMA extension for scoping reviews (PRISMA-ScR): checklist and explanation. Ann Intern Med 2018;169:467-73.

34 Kowatsch T, Otto L, Harperink S. A design and evaluation framework for digital health interventions. it - information technology. 2019: 61, 253-63.

35 Daudt HML, van Mossel C, Scott SJ. Enhancing the scoping study methodology: a large, inter-professional team's experience with Arksey and O'Malley's framework. BMC Med Res Methodol 2013;13:48.

36 World Health Organization. Classification of digital health interventions 1.0 : a shared language to describe the uses of digital technology for health, 2018. Available: https://www.who.int/ reproductivehealth/publications/mhealth/classification-digital-healthinterventions/en/ [Accessed 11 Jun 2020].

37 Hong QN, Pluye P, Bujold M, et al. Convergent and sequential synthesis designs: implications for conducting and reporting systematic reviews of qualitative and quantitative evidence. Syst Rev 2017;6:61. 